\section{Check for updates}

Cite this: J. Anal. At. Spectrom., 2019, 34,1033

DOI: 10.1039/c9ja90023c

www.rsc.org/jaas

\title{
Retraction: Non-matrix matched calibration of element contents in stainless steel by using nanosecond and femtosecond laser ablation inductively coupled plasma mass spectrometry
}

\author{
Jiuling Meng, ${ }^{a}$ Xiaocheng Zhang, ${ }^{b}$ Jingrui Cao, ${ }^{\mathrm{b}}$ Yupeng Zhao ${ }^{\mathrm{b}}$ and Tao Lüab
}

Retraction of 'Non-matrix matched calibration of element contents in stainless steel by using nanosecond and femtosecond laser ablation inductively coupled plasma mass spectrometry' by Jiuling Meng et al., J. Anal. At. Spectrom., 2019, DOI: 10.1039/c8ja00443a.

We, the named authors, hereby wholly retract this Journal of Analytical Atomic Spectrometry article as we did not have permission to publish this work and the findings are unreliable.

The authors did not have permission to publish this work. Most of the original experimental data, which is significant to this work was collected by a M.S. student and her supervisor who were not listed as co-authors on the paper. The manuscript was submitted and revised without their permission.

In addition, some of the reported findings are unreliable. Fig. 7 presents the time-sequence snapshots of the laser ablation plumes of GSE-1G and GBW01395 standard samples. However, the experimental conditions are not the same as the experimental conditions for LA-ICP-MS analysis of alloy steel. The laser wavelength for the snapshot experiment was not the same as that for microanalysis. The former experiment has been performed in air when one laser pulse with $355 \mathrm{~ns}$ wavelength and $25 \mathrm{~mJ}$ energy, while the latter experiment has been done in inertia gas He when one laser pulse with $193 \mathrm{~nm}$ or $257 \mathrm{~nm}$ wavelength and $3.5 \mathrm{~J} \mathrm{~cm} \mathrm{~cm}^{-2}$ or $3.64 \mathrm{~J} \mathrm{~cm}^{-2}$. Therefore, the explanation of these results is unreliable. Furthermore, the snapshots of another steel has replaced the experimental results of GBW01395, though their characteristics are very similar. As a result, the explanation of ablation plumes is unreasonable and unbelievable.

Finally, the major conclusion of this manuscript that "techniques such as fs-LA-ICP-MS and non-matrix matched calibration can successfully determine trace element content in alloy steel" is questionable and invalid.

Signed: Jiuling Meng, Xiaocheng Zhang, Jingrui Cao, Yupeng Zhao, Tao Lü

Date: $1^{\text {st }}$ April 2019

Retraction endorsed by Jeanne Andres, Executive Editor, Journal of Analytical Atomic Spectrometry

${ }^{a}$ State Key Laboratory of Geological Process and Mineral Resources, School of Earth Sciences, China University of Geosciences, Wuhan 430074, China

${ }^{b}$ School of Mathematics and Physics, China University of Geosciences, Wuhan, 430074, China. E-mail: lvtaohn@sina.com 